\title{
Twitter User Modeling based on Indirect Explicit Relationships for Personalized Recommendations
}

\author{
Abdullah Alshammari, Stelios Kapetanakis, Nikolaos Polatidis, Roger Evans, Gharbi \\ Alshammari \\ School of Computing, Engineering and Mathematics, University of Brighton, Brighton, United \\ Kingdom \\ (A.Alshammari1, S.Kapetanakis, N. Polatidis, R.P.Evans, \\ g.alshammari) @brighton.ac.uk
}

\begin{abstract}
Information overload has increased due to social network website use in recent times. Social media has increased the popularity of websites such as Twitter. It is believed that a rich environment is provided through Twitter whereby information sharing will be able to aid in recommender system research. This paper will focus upon Twitter user modeling through the utilization of indirect explicit relationships that exist amongst users. The further aim of this paper is to ensure that personal profiles are built via the use of information that will be sourced from Twitter so as to provide recommendations that are more accurate. The proposed method adopts Twitter user's indirect explicit relationships in order to get information which is vital in the process of building personal user profiles. The proposed method has been validated through the implementation of an offline evaluation using real data. Proposed user profiles' performances have been compared with each other and against the baseline profile. The performance of this has been validated using real data and is both practical and effective.
\end{abstract}

Keywords: Recommender systems, User modeling, User profiling, Explicit relationships, Twitter.

\section{Introduction}

Real-time web is rising as a technology or platform that enables users to share information and communicate in different contexts like Twitter, which is one of the most popular micro-blogging platforms today [16]. It is used by millions of people around the world. Through this platform, users are able to share short messages, up to 280 characters in length, referred to as tweets [16]. It is a very popular means of sharing information as well as effectively reaching a wide audience. It can also be considered as a distinctive shaper of social network platforms because it presents relationships between users based on a review strategy. This platform makes it very different in comparison to other reciprocal social networking platforms such as Facebook. Relationships between Twitter users can either be informational (i.e., reading news) or social (i.e., interacting with friends). Also, users can follow other users in order to receive informational posts [1]. The features of Twitter make it useful as a major source for the 
modeling of users involved in networks characterized by interactions and relationships $[1,17]$.

Researchers in [1] have demonstrated that Twitter is an important resource in regard to recommender systems, which are a powerful and integral part of the web as well as mobile applications. Such systems do have a primary objective, which is to ensure that context-aware, personalized, and real-time information is provided in order to raise sales as well as user satisfaction. Numerous researchers have exploited Twitter for modeling users, building user profiles, and recommending items in an accurate way such as building a profile based upon user tweets to recommend useful news. The usual way of building such a profile lacks up-to-date information about users' interests. The authors in [4] have solved this problem by using friends' recent tweets to enhance the user's profile with up-to-date information.

This paper is focused on modeling Twitter users through the exploitation of relationships with the aim of enhancing recommendations in the recommender systems. It is an extension of the previous work of the researchers of this paper [4] which was focused on direct explicit relationships (friends' accounts only), whereas this paper focuses on indirect explicit relationships. Indirect explicit relationships contain accounts that the user receives in their tweets from outside the friends list, such as friends of friends. Proposed profiles will contain tweets from these accounts for a period of 2 weeks (short-term profiles) to make sure that profiles contain recent activity that reflects users' interests. The methodology will contain a user profile designed or built from accounts that are outside the user's friends list. The proposed model makes use of the influence rule put forth in the authors' previous work [4], which will be explained generally in the following section. The influence rule will be used for the identification of influential friends. Therefore, their tweets will be used in the building of the user profile via an examination of each tweet received within the last two weeks (short-term). In light of the presented methodology, the following suppositions are made:

- We propose a method that builds user profiles from the tweets of accounts that have indirect explicit relationships with the user.

- The proposed method has been experimentally evaluated using a real dataset, wellknown metrics, and against a baseline; thus, the recommendations delivered are more accurate compared to the baseline.

The rest of the paper is constructed as follows: Section 2 delivers the related work, section 3 presents the proposed method, section 4 explains the experimental evaluation and section 5 contains the conclusions.

\section{Related work}

A method proposed by the authors in [11] was utilized so that a recommender system is designed based on tweets from Twitter to ensure that the accuracy of recommended news articles is improved. In order to build the user profile, the nouns were extracted from the user's tweets as well as retweets. The results indicated that the recommendations (Twitter activity-based) seem to be more accurate when compared to random 
recommendations. The authors in [8] also proposed TRUPI system that combined the user's tweets history with social features. Also, the system tried to identify the dynamic interest of the user on various topics and to measure how the interest of the user will change over a given period of time.

Temporal dynamics (in profiles of Twitter) were analysed by the researchers in [1] to understand personalized recommendations. The profiles that they built were based on entities (celebrities and places) and hashtags. Variables that were taken into consideration are enrichments (use of external resources like Wikipedia) and user's activity. Results revealed that entity-based profiles, which were built through the use of shortterm time (recent activities) as well as enrichment, perform much better compared to other profile types. However, there is a problem in that a number of users lack sufficient data to enable the creation of reliable user profiles. In the research of [12], the authors identified that applying a decay function on long-term profiles, which gives more weight value to recent or newer topics than older topics of interests, showed much better performance in delivering recommendations when compared to long-term profiles not using this function. Also, the researchers in [2] have previously shown that short-term profiles are superior to complete profiles.

A major solution can be enacted through the enrichment of a user profile with other data. Twitter user profiles were modeled by the authors in the study of [2] using various dimensions. Each of their qualities was compared, including the enrichment dimension. The outcomes indicated that utilizing external resources, news articles for instance, seem to be much better than compared to reliance only on Twitter data.

User profile enrichment has been carried out in various ways. These can be done using textual external resources or by exploiting tweets' URLs. Through the exploitation URLs from tweets, a Cat stream system was proposed by [9] which used traditional classification techniques in profiling users of Twitter. It concentrated only on URLs attached in tweets. However, this system is not appropriate for users who do not provide a sufficient number of tweets that contain URLs. A group of tweets was categorised by the authors in [3] as interesting or uninteresting. This categorisation was done through the use of crowd sourcing. This method showed that a URL link's existence is a feature which is successful when it comes to the selection of high accuracy interesting tweets. This factor has its own shortcoming, in that it may classify a tweet incorrectly when linked with un-useful content. Also, users normally do not post enough URLs in their tweets [10].

In regard to the external resources technique, it has been shown by $[1,9]$ that profiles that included external resources outperformed those profiles which were built using just Twitter activity. As a result, there is an adequate supply of user profiles with more information, which can lead to an improvement in the recommender system accuracy. However, there is data gathered from external resources that will not be relevant to the interest of the user. This can negatively affect the recommender system's performance.

The relationship which exists amongst Twitter users is a field that is yet to be studied. Such research can help to ensure that a user is characterized, thereby improving the recommender system's performance based on profiles enriched by the tweets of others who participate in these relationships. It is very clear that any single user who tends to produce short-text data (retweets and tweets) can be modelled based on his behaviour 
and history through a collection of historical data (also known as timeline) that has been generated by the user himself. However, in order to provide enough information for building profiles, such a method needs a design system that reaches deeper into the past for data collection. Moreover, the collected information might be too out of date to be used in profiling as users' interests change. Also, the recent activities of most users do not have sufficient data or URL data to enrich their profiles from them. To address the problem of data shortage, the authors in [4] showed that direct explicit links between users should be used for the purpose of enriching the user profile. This finding will help expand the recent activities that are relevant. This method has an advantage, which is that profiles are built from activities that are more recent. Through this method, the recommender system's performance, which is based on these tweets, will be improved. This paper continues the exploration of these links between users within a network to improve the profile through exploiting indirect explicit relationships.

In order for the following links to be exploited, influential users will be gleaned from the user's friends of friends. The majority of research focuses on the influence of a particular user, which is based on that users popularity. This rate is indicated by the number of friends and followers of the user. How a user's interaction with others serves as an influence was also clarified in previous researches $[5,6,7,18]$. Additionally, various Twitter influence measures were collected as well as classified from the literature in [13]. These studies explained that trying to deem someone as influential in a social network is a conceptual problem. They further stated that there is no agreement regarding who or what is an influential user. Subsequently, the authors in [4] argued that there is a need for creating a method which will be able to generate or produce an influence score based on the perspective of the normal user in relation to his or her interaction and behaviour. Their proposed influence score was higher when it identified influential friends of the user and then utilized those friends as a valuable source for building the user profile. As a result, more accurate recommendations were delivered.

\section{$3 \quad$ Proposed Methodology}

Generally, the recommender system consists of two phases: (1) user profiling and (2) item ranking. User profiles here are constructed as an extended version of our previous work [4]. Moreover, more resources will be identified through the indirect explicit relationships. The user's tweets will then be extracted and added to the profile. After that, and based on the user profile, recommendation items are ranked.

The general framework of our proposed approach is shown in Figure 1. By using the Twitter API, the user's data is collected and then processed in order to identify influential friends and build the primary profile (STBLCinf) based on important keywords written by the user and his friends. This step is the basic idea in the previous work [4], and the proposed profiles in this approach will be an extended version of the STBLCinf profile. The steps of each phase will be explained in detail in the following subsections. 


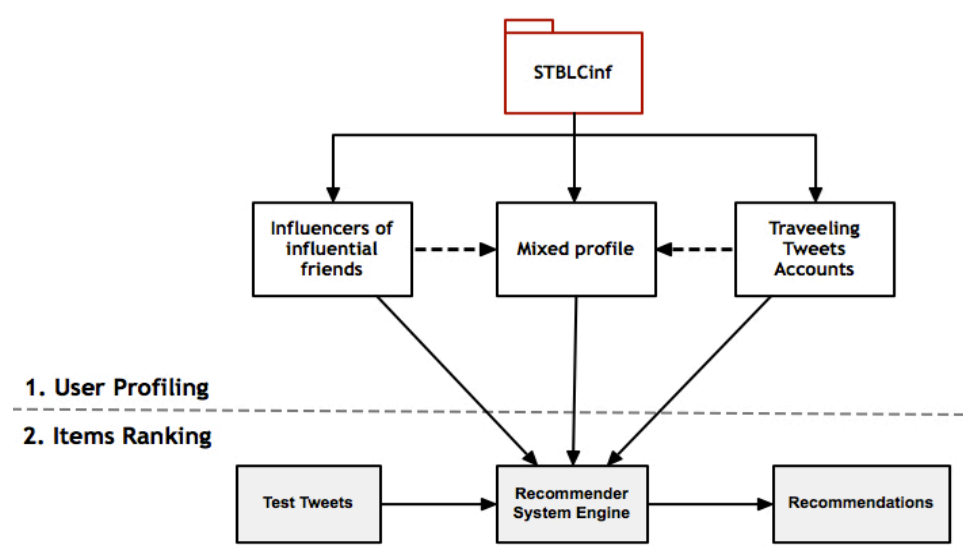

Fig. 1. The main stages of the proposed approach.

\subsection{User Profiling}

In this phase and in general, tweets that are posted by other users, those who have explicit relationships with the user (i.e., friends), are used in building the profiles. All profiles are built and represented by keywords (bag of words), while pre-processing is applied in order to filter tweets by extracting only important content. Tweets posted and re-tweeted by the user shows the user's interests. On the other hand, tweets received and collected via explicit relationships through the following of links require examination and classification in order to include them in the profiles.

In our previous work [4], the proposed influence score algorithm was able to identify the influential friends of the user and their tweets within a brief time, which are used in building the profile (STBLCinf profile). By exploiting the indirect explicit relationships, two different sources were used in this paper to build the user profile from namely influential friends of influential friends and accounts of travelling tweets. These profiles will be an extended version of the STBLCinf profile.

In inf of inf source and for each user in dataset, the timeline tweets, favourited tweets, and friends list are collected for each influential user in the friends list. Additionally, this information is collected for each influential friend. Then, the influence score is computed between the user and his friends in order to rank his friends based on their influence score and to identify those that are influential. Next, friends are clustered into three categories based on the K-means algorithm: influential, less influential, and noninfluential friends. The tweets of influential friends will be added to the profile. While the tweets of non-influential friends will be excluded. Tweets of less influential friends will be classified into representative (re-tweetable) or not representative (not retweetable). As in our previous work in [4], the trained classifier that has the highest accuracy in the training set, and was used to classify tweets before, is used here to classify the tweets of less influential friends. Furthermore, various classifiers were used in training the labelled dataset that is built from the user's tweets. Thus, the tweets classified as representative (re-tweetable) are added to the tweets in the profile. 


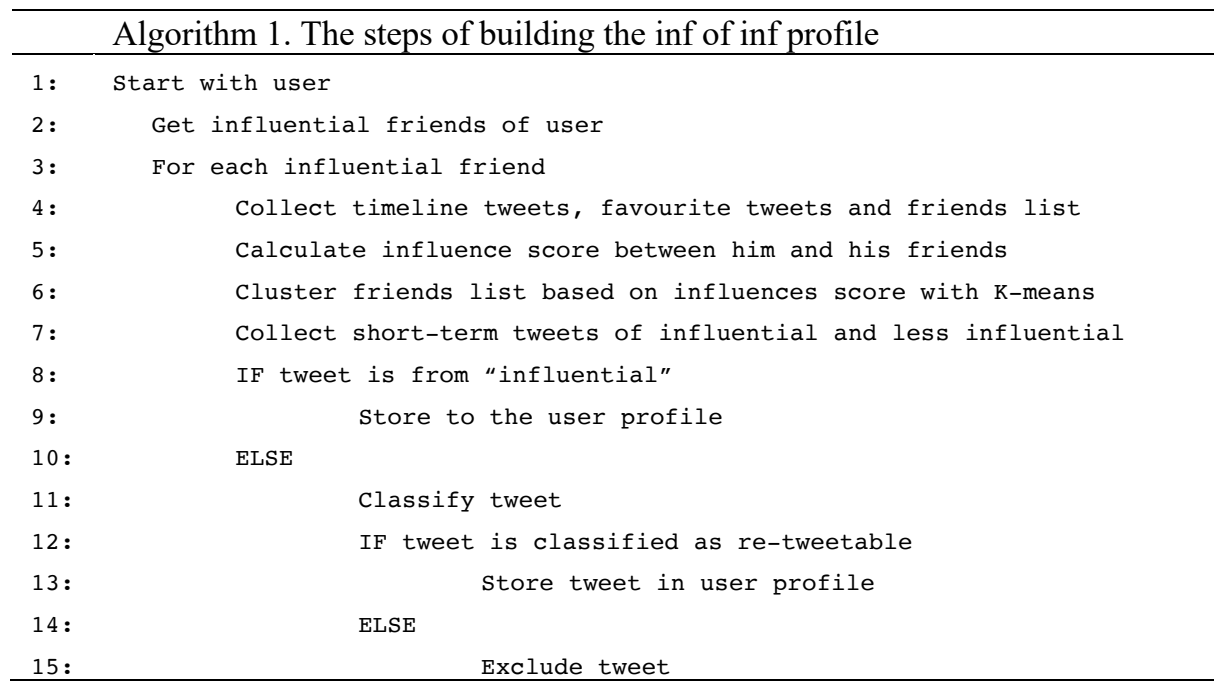

In the second source, travelling tweets accounts (TTA) are identified. This is done by extracting the accounts that appeared in the user timeline but are not in the user's friends list. Moreover, the user showed some interests by re-tweeting their tweets. After that, tweets from these accounts are collected within brief timespan. The same classifier is used here for classifying tweets into representative (re-tweetable) or not representative (not re-tweetable). The former tweets will be included in the user profile. By the end of this stage, three profiles are built in order to measure their efficiency in the recommender system, which are: inf of Inf, TTA, and the mixed profile (inf of inf + TTA).

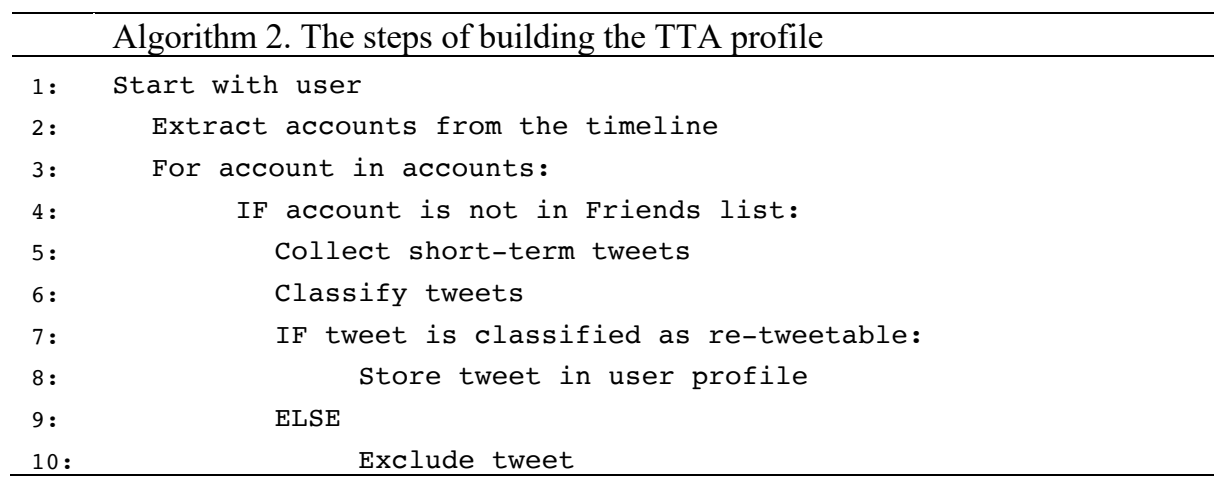

\subsection{Items Ranking}

Recommendation items in this stage will be represented by those tweets which the user has shown interest in by retweeting. The model which will be used is Vector space 
representation since it will take recommendation items and the user profile as vectors. The angle which exists between them will then be computed.

In order to rank the various groups of tweets, user profiles that have been developed as explained in the former subsection will be used. All tweets inside the recommendation items will need to be evaluated based on how similar they are to user's profiles. Tweets will be excluded whenever the text contains less than 3 words. Lastly, the similarity score is computed through cosine similarity equation. Therefore, every recommendation item is ranked, and then the recommendation tweets are delivered to user.

\section{$4 \quad$ Experimental Evaluation}

For our proposed method's advantages to be validated, there is the implementation of the tweets recommender system. There was also the performing of the offline evaluation using some users. By using Twitter API, which can be found on the website's development section, the timelines of 40 users chosen at random were collected and examined. The dataset, which contains the 40 users, is balanced in terms of activity (active and less active users) and engagement (tweeters and re-tweeters). In the recommendation items, test tweets are collected from the user's timelines based on actions of interests that the user showed via re-tweeting. The following section explains the mechanism of collecting test tweets.

\subsection{Experiment Setup}

When the information is collected from the examined user's timeline, the dataset is split into three-time frames as it was done in previous work [4].

The tweets that are in the first timeframe (the last 2 weeks) will be used as test items (test tweets). The tweets that are from the second timeframe (between 2 and 4 weeks prior) will be used in building profiles from the mentioned resources. The third timeframe (more than 4 weeks prior) will be used alongside the second timeframe for computing the influence score generated from the influential friends' timelines. Additionally, information gathered from the timeline of the user during the set timeframe will be used in the machine learning classifiers.

Profiles: In this experiment, three profiles will be built, compared against each other, and compared against the profile created in the previous research [4] of the authors. All profiles were built within short-time frame. The profiles are:

- The influential tweets of friends (STBLCinf): This profile includes the timeline tweets, influential tweets, and less influential tweets that are classified as representative (re-tweetable). This profile is considered as the basis of our proposed method because the other profiles are extended versions of this one.

- Influential of influential (inf of inf): This profile includes all tweets from STBLCinf, tweets of influential friends of influential friends, and those classified as less influential friends of influential friends' tweets, which are considered as representative. 
- Travelling Tweets Accounts (TTA): This profile contains all tweets from STBLCinf and the tweets from accounts of travelling tweets that are classified as representative.

- Mixed profile (inf of inf + TTA): this profile contains tweets of STBLCinf, inf of inf, and TTA.

Test tweets: will be used for evaluating how accurate the recommender system is based on each proposed profile. They are collected from the first timeframe (the last 2 weeks) as clarified previously, and they will be utilized as recommendation items. Each tweet in the test tweets can be relevant or non-relevant. Relevant tweets are tweets that the user re-tweeted whereas the non-relevant tweets are tweets that the user did not show interest in by retweeting.

Evaluation metrics: Offline evaluation has been used in measuring the accuracy of the recommender system using various profiles [15]. The built user profiles will be plugged into the same recommender system with the same test tweets in order to compare their performance. The metrics used in this study for measuring the system's performance accuracy are average of precision @ $\mathrm{k}(\mathrm{P} @ \mathrm{k})$, average precision (AP), and mean average precision (MAP) [14]. Average of precision is used in measuring how good the system is in retrieving relevant items @ $\mathrm{k}$, and MAP is used in measuring how useful the system is for retrieving all items that are relevant in a good order. P@k is the amount of correct recommendations in the top- $n$ list of recommendations and is defined in equation 1. AP is defined in equation 2 where $\mathrm{p}(\mathrm{k})$ is the precision $@ \mathrm{k}$ and rel $(\mathrm{k})$ is an indicator counting as 1 when the item is relevant or zero. Moreover, items not retrieved, but considered relevant, receive a score of zero. Lastly, MAP, which is defined in equation 3, has $\mathrm{Q}$ being a query and the equation returning the mean of the average precision scores for a set of queries.

$$
\begin{gathered}
P @ k=\frac{\text { relevant recommended items }}{\text { total recommended items }} \\
A P=\frac{\sum_{k=1}^{n}(p(k) * \text { rel }(k))}{\text { number of relevant recommendations }} \\
M A P=\frac{\sum_{q=1}^{Q} A P(q)}{Q}
\end{gathered}
$$

\subsection{Results}

In this subsection, the profiles that were built from tweets, and derive from different resources of the indirect explicit relationships of the examined user, will be compared against each other and the profile mentioned from the previous research in [4], which is the STBLCinf. The purpose of this step is to be able to determine which profile performs better and to ascertain the required scope for collecting relative tweets. Results 
from this stage have already been presented in the previously mentioned research. The tested values of $\mathrm{k}$ are from 1 to 10 . In the metrics of the $\mathrm{P} @$ top-k recommendations, when the TTA and Inf of Inf were compared, the results show that the Inf of Inf profile outperformed the TTA profile in all P@top-10 recommendations. When both were compared to the mixed profile, which is TTA + Inf of Inf, results showed that the Inf of Inf profile outperformed the others 9 times in the top 10 recommendations. In contrast, the performance of the mixed profile was better than the TTA profile because it achieved a higher AP value 5 times at $\mathrm{k}=1,3,8,9$ and 10. Moreover, the TTA profile achieved a higher AP value in only 3 instances. When all the profiles were compared against the baseline, which is STBLCinf, the results showed that the Inf of Inf profile performed better than other profiles, especially STBLCinf. In contrast, its performance was better in 5 of the top 10 recommendations whereas the STBLCinf was better 3 times. (See Figure 2a).

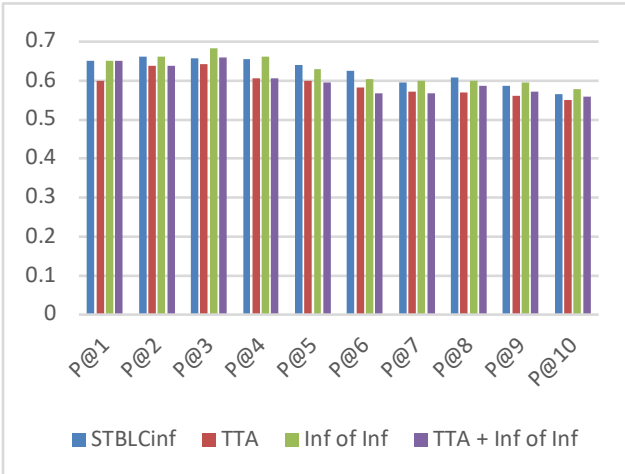

(a) Average of Precision @ $\mathrm{k}(\mathrm{P} @ \mathrm{k})$

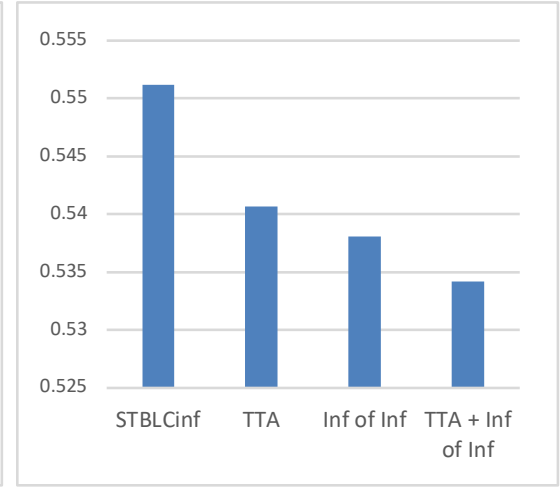

(b) Mean Average Precision (MAP)

Fig. 2. Average of precision $@$ k from 1 to 10 and the Mean average precision (MAP) of the profiles.

In the metric of MAP, the profiles were compared in order to see their overall performance in recommending all relative items. The results showed that the TTA profile achieved a higher MAP value than the Inf of Inf profile by $0.2 \%$. The mixed profile achieved less MAP value than the TTA by $0.6 \%$ and less than the Inf of Inf by $0.4 \%$. On the other side, the STBLCinf performance was compared to the profiles, and the results showed that it performed better than the TTA, Inf of Inf, and the mixed profile by $1 \%, 1.2 \%$, and $1.6 \%$ respectively. (See Figure $2 b$ ).

\subsubsection{Dividing dataset based on activity:}

The dataset is divided based on activity in order to see deeper analysis and to understand the results in an advanced way. The activity (engagement) of each user was computed using the equation below proposed by [16], which calculates how active a user has been on Twitter since joining.

$$
\text { Engagement }(\text { Activity })=\frac{\text { Tweets }+ \text { Retweets }+ \text { Replies }+ \text { Favourite }}{\text { Account_Age }}
$$


After calculating the activity, users in the dataset are ranked in ascending order based upon their activity scores. Then, they are divided into active and less active, each division having 20 users. In the less active user group, and using the metric of P@top-10 recommendations, Figure $3 \mathrm{a}$ shows that the Inf of Inf profile outperformed the TTA and the TTA + Inf of Inf profiles 9 out of 10 recommendations. In comparison to the STBLCinf profile, the Inf of Inf profile achieved a higher AP value in 3 instance whereas the former achieved a better performance score in 4 instances. In comparison, the mixed profile was slightly better than the TTA profile in delivering related items in the top 10 recommendations. Also, both profiles achieved the lowest performance. In the MAP metric, the result was similar to the general evaluation before the dataset was divided. It showed that the profiles TTA, Inf of Inf, and TTA + Inf of Inf achieved a lower MAP value than the STBLCinf by $1.6 \%, 2.6 \%$, and $3.1 \%$ respectively. (See Figure $3 b$ ).

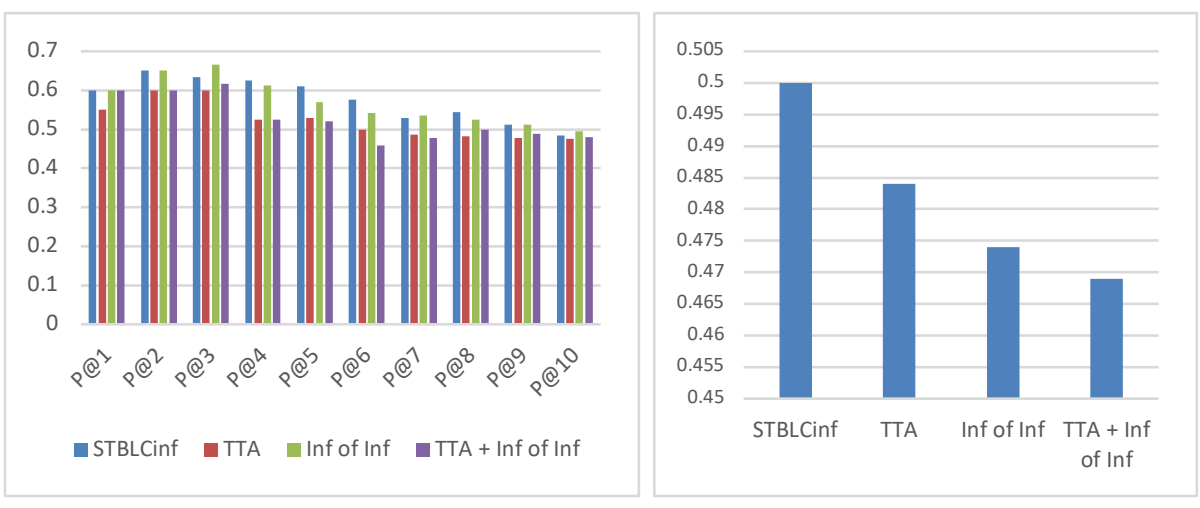

(a) Average of Precision @k (P@k)

(b) Mean Average Precision (MAP)

Fig. 3. Average of precision $@ \mathrm{k}=1-10$ and the Mean average precision of the profiles of less active users in the dataset.

In the Active user group, the results clarified that the Inf of Inf profile outperformed all other profiles in 4 instances (See: Figure 4a). Surprisingly, the STBLCinf profile did not outperform all the profiles in any of the $\mathrm{k}$ values. The TTA profile achieved the lowest AP in 4 instances. When the top 10 recommendations were delivered, results proved that the Inf of Inf profile was able to deliver related recommendations to the active users more accurately than the other profiles. In the MAP metric, surprisingly, the Inf of Inf profile achieved the highest value. Moreover, it was better than the STBLCinf. However, the TTA and TTA + Inf of Inf profiles achieved lower MAP values. This may prove that the Inf of Inf profile is more suitable for the active users than the less active users. (See Figure 4b). 


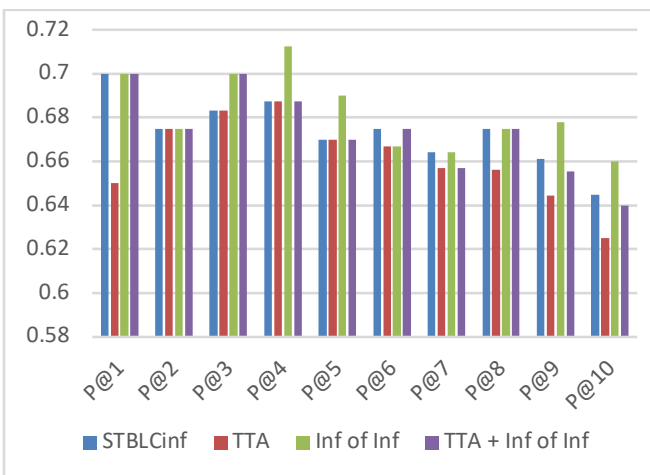

(a) Average of Precision @ $\mathrm{k}(\mathrm{P} @ \mathrm{k})$

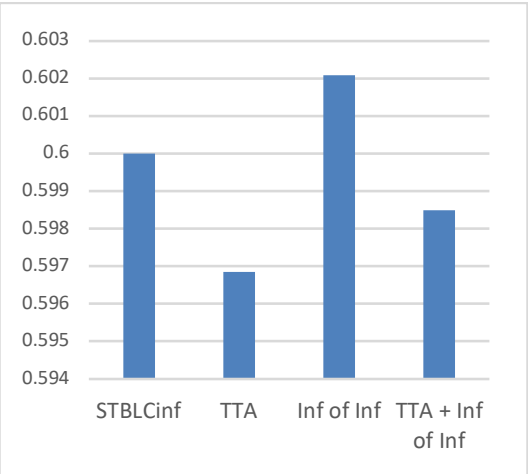

(b) Mean Average Precision (MAP)

Fig. 4. $\mathrm{P} @ \mathrm{k}=1-10$ and MAP of the profiles of active users in the dataset.

\section{Conclusion}

In conclusion, this research proposed a new technique for building a user's profile based on exploiting the Twitter explicit links structure with the aim of improving the performance of recommender systems that are based on tweets. This innovative profile takes advantage of the links between a user and his or her friends in order to gather a tweets set within a brief timeframe and to utilize them in profile building. Moreover, the redefined influence rule in our previous work helped us to cluster friends of influential friends into three categories: influential, less influential, and non-influential. Consequently, tweets of influential users were added directly to the profile while tweets of non-influential users were excluded. Tweets of less influential users were classified, and the re-tweetable tweets were included in the created profile. An offline evaluation experiment was done by using a tweets-recommender system, which was used to validate the effectiveness of this proposed technique. Its effectiveness has been shown by comparing and testing it against the baseline profile built from the direct explicit relationships (STBLCinf). Therefore, the profile developed through proposed method was able to outperform the other profiles in recommending the top 10 recommendations. Also, it was the best performing profile with active users. In future work, more exploration can be done to discover through the use of explicit relationships more relationship types that exist among a user and his or her friends. Additionally, we would like to further investigate the similarity that is exists between a user and his or her followers in order to extend the tweets set that forms the interests of the user.

\section{References}

1. Abel, F., Gao, Q., Houben, G. J., \& Tao, K. (2011, June). Analyzing temporal dynamics in twitter profiles for personalized recommendations in the social web. In Proceedings of the 3rd International Web Science Conference (p. 2). ACM. 
2. Abel, F., Gao, Q., Houben, G. J., \& Tao, K. (2013, August). Twitter-Based User Modeling for News Recommendations. In IJCAI (Vol. 13, pp. 2962-2966).

3. Alonso, O., Carson, C., Gerster, D., Ji, X., \& Nabar, S. U. (2010, July). Detecting uninteresting content in text streams. In SIGIR Crowdsourcing for Search Evaluation Workshop.

4. Alshammari, A., Kapetanakis, S., Evans, R., Polatidis, N., \& Alshammari, G. (2018). User Modeling on Twitter with Exploiting Explicit Relationships for Personalized Recommendations. Paper presented to the 18th International Conference on Hybrid Intelligent Systems. Porto. 13-15 December.

5. Anger, I., \& Kittl, C. (2011, September). Measuring influence on Twitter. In Proceedings of the 11th International Conference on Knowledge Management and Knowledge Technologies (p. 31). ACM.

6. Bakshy, E., Hofman, J. M., Mason, W. A., \& Watts, D. J. (2011, February). Everyone's an influencer: quantifying influence on twitter. In Proceedings of the fourth ACM international conference on Web search and data mining (pp. 65-74). ACM.

7. Chen, C., Gao, D., Li, W., \& Hou, Y. (2014, July). Inferring topic-dependent influence roles of Twitter users. In Proceedings of the 37th international ACM SIGIR conference on Research \& development in information retrieval (pp. 1203-1206). ACM.

8. Elmongui, H. G., Mansour, R., Morsy, H., Khater, S., El-Sharkasy, A., \& Ibrahim, R. (2015, April). TRUPI: Twitter recommendation based on users' personal interests. In International Conference on Intelligent Text Processing and Computational Linguistics (pp. 272-284). Springer, Cham.

9. Garcia Esparza, S., O'Mahony, M. P., \& Smyth, B. (2013, March). Catstream: categorising tweets for user profiling and stream filtering. In Proceedings of the 2013 international conference on Intelligent user interfaces (pp. 25-36). ACM.

10. Karidi, D. P., Stavrakas, Y., \& Vassiliou, Y. (2016, July). A personalized Tweet recommendation approach based on concept graphs. In Ubiquitous Intelligence \& Computing, Advanced and Trusted Computing, Scalable Computing and Communications, Cloud and Big Data Computing, Internet of People, and Smart World Congress (UIC/ATC/ScalCom/CBDCom/IoP/SmartWorld), 2016 Intl IEEE Conferences (pp. 253260). IEEE.

11. Lee, W. J., Oh, K. J., Lim, C. G., \& Choi, H. J. (2014). User profile extraction from Twitter for personalized news recommendation. Proc. 16th Advanced Communication Technology, 779-783.

12. Piao, G., \& Breslin, J. G. (2016, September). Exploring dynamics and semantics of user interests for user modeling on Twitter for link recommendations. In Proceedings of the 12 th International Conference on Semantic Systems (pp. 81-88). ACM.

13. Riquelme, F., \& González-Cantergiani, P. (2016). Measuring user influence on Twitter: A survey. Information Processing \& Management, 52(5), 949-975.

14. Shani, G., \& Gunawardana, A. (2011). Evaluating recommendation systems. In Recommender systems handbook (pp. 257-297). Springer, Boston, MA.

15. Uysal, I., \& Croft, W. B. (2011, October). User oriented tweet ranking: a filtering approach to microblogs. In Proceedings of the 20th ACM international conference on Information and knowledge management (pp. 2261-2264). ACM.

16. Vosoughi, S. (2015). Automatic detection and verification of rumors on Twitter (Doctoral dissertation, Massachusetts Institute of Technology).

17. Weng, J., Lim, E. P., Jiang, J., \& He, Q. (2010, February). Twitterrank: finding topic-sensitive influential twitterers. In Proceedings of the third ACM international conference on Web search and data mining (pp. 261-270). ACM. 